\title{
Selective Route Based on SNR with Cross-Layer Scheme in Wireless Ad Hoc Network
}

\author{
Istikmal, Adit Kurniawan, and Hendrawan \\ School of Electrical Engineering and Informatics, Institut Teknologi Bandung, Jl. Ganesha 10, Bandung 40132, Indonesia \\ Correspondence should be addressed to Istikmal; istikmal@telkomuniversity.ac.id
}

Received 2 December 2016; Revised 6 March 2017; Accepted 13 April 2017; Published 25 May 2017

Academic Editor: Sandra Céspedes

Copyright (C) 2017 Istikmal et al. This is an open access article distributed under the Creative Commons Attribution License, which permits unrestricted use, distribution, and reproduction in any medium, provided the original work is properly cited.

In this study, we developed network and throughput formulation models and proposed new method of the routing protocol algorithm with a cross-layer scheme based on signal-to-noise ratio (SNR). This method is an enhancement of routing protocol ad hoc on-demand distance vector (AODV). This proposed scheme uses selective route based on the SNR threshold in the reverse route mechanism. We developed AODV SNR-selective route (AODV SNR-SR) for a mechanism better than AODV SNR, that is, the routing protocol that used average or sum of path SNR, and also better than AODV which is hop-count-based. We also used selective reverse route based on SNR mechanism, replacing the earlier method to avoid routing overhead. The simulation results show that AODV SNR-SR outperforms AODV SNR and AODV in terms of throughput, end-to-end delay, and routing overhead. This proposed method is expected to support Device-to-Device (D2D) communications that are concerned with the quality of the channel awareness in the development of the future Fifth Generation (5G).

\section{Introduction}

Ad hoc network is expected to have a more significant role in the implementation of future 5G. Ad hoc network also provides the inclusion of $\mathrm{D} 2 \mathrm{D}$ communication, a technology which becomes one of the key enablers for several advance communication techniques such as critical communication [1]. Ad hoc network also supports the development of Mobile-to-Mobile (M2M) communication [2, 3]. In D2D and M2M communication, the successful data transmission is influenced by the mechanism of the routing protocol. This protocol is responsible for seeking routes as the delivery path and has to be able to adapt to different situations, such as the movement of the users and the channel quality. Various routing mechanisms have been proposed for D2D $[4,5]$ but are restricted to the route discovery efficiency due to the fully distributed network [6]. The keys to improve the efficiency in route discovery are reducing the overhead and finding the route with good quality connection to improve the throughput performance.

Efficient communication must offer the capability to support application with rapid bandwidth requirement [7]. With this requirement, the path to deliver the data should have good quality. Moreover, to achieve intelligent D2D communication with resource constraint and intermittent connectivity, the devices require intelligent protocols which the conventional routing protocols cannot solve [8].

The conventional routing protocol that uses hop count as a metric for determining the path is perceived no longer appropriate for applications that require a good quality communication path. The protocol should be more adaptive and has to consider the link quality being used as the path to deliver the data $[9,10]$. The routing algorithm that helps network find a reliable path will improve the network resilience against path break and packet loss. The cross-layer scheme is one of promising methods that has become popular in recent research to achieve maximum network utility [11]. This method shares information between layers and joint cooperations to increase network performance with general or specific solutions.

Previous studies had proposed some new methods with more attention to the quality of path. These methods were based on the quality of service (QoS) and the quality of the channel (QoC). In the case of QoS, one of such research was quality awareness with effective estimated throughput [12]. Another related research is a mechanism to find high 
throughput path by taking the expected total number of Medium Access Control- (MAC-) layer transmissions into account [13]. This research is further enhanced using cooperative transmission [14]. On the other hand, [15] and [16] proposed a routing metric that considered the expected transmission time and high throughput route selection with medium time metric, respectively. In the case of QoC, a mechanism to improve multihop routing using SNR by adding the SNR information into Route Request (RREQ) was proposed in $[9,17]$. The use of SNR and Receive Power (RP) to find the route was proposed by $[18,19]$ by adding up the SNR information into Route Reply (RREP) mechanism. In [20] and [21], routing selection was improved using minimum summation of inverse SNR and weighted SNR average of all links in the path, respectively. Moreover, a study reported in [22] proposed a routing protocol for wireless sensor networks through SNR-based dynamic clustering, while that reported in [23] used cooperative Automatic Repeat reQuest (ARQ) system to minimize total network energy consumption while delivering a minimum required SNR. Routing protocol based on streamlines of potential field where data rate depended on SNR was studied in [24], and cluster routing protocol considering the SNR and residual energy of the nodes with cross-layer design was proposed in [25]. However, previous work that used SNR in routing protocols had some issues in the methods. To solve these issues, we proposed new methods. Details on the issues and the proposed method are discussed in Sections 2 and 4, respectively.

The paper is organized as follows. Section 2 describes the related work and the contributions of this research. Section 3 describes the network model and throughput formulation. Section 4 describes the weakness of earlier methods and the design of selective route based on SNR with crosslayer scheme. Section 5 describes the routing protocol performance and analysis. Finally, Section 6 is the conclusion and future research opportunities.

\section{Related Work}

A mechanism to improve multihop routing using SNR by adding the SNR information into RREQ was proposed in [17]. This scheme used the average route quality as the ratio between the overall path quality and the number of hops. The result showed improvement in network throughput and reduced packet error rate. However, adding SNR information in the broadcast of RREQ mechanism had caused increasing overhead and complexity of the route discovery process.

Study in [9] discussed the important of SNR as link quality to use in routing protocol. The paper suggested crosslayer mechanism with SNR information to obtain better routing. This mechanism added SNR information in each RREQ packet; the destination node then determined the best route and replied by sending a RREP packet. Since the RREQ and the RREP frame carried the new extension field, this method had caused higher overhead and complexity in both way mechanisms.

Authors in [18] used SNR and RP as valuable information for routing protocol. The proposed scheme added two extra fields in RREP packet to store SNR and RP information. The source node selected the route based on any best available values of SNR or RP. The performance results with cross-layer design improved their performances with respect to service quality.

The same method with addition of SNR information in RREP mechanism was proposed by [19]. In this method; the authors applied lower to upper layers scheme, where SNR information from physical layer was utilized in routing protocol. From the simulations, the proposed method achieved higher throughput than the conventional routing protocol that used hop count as routing metric. In [20], routing selection was improved using minimum summation of the inverse SNR. The metric was equivalent to this method of all the links in the path. Through simulation, the proposed method claimed gain for better performance in terms of amount of delivered packet and end-to-end delay. Although this method is used to find the path with good channel quality, the selection of the path with this method may have one or more links with very low quality.

Authors in [21] used SNR as channel-aware routing metric. The proposed scheme utilized the weighted signalto-noise ratio average. The best route was selected based on the minimum accumulated SNR average. The algorithm had resulted in higher throughput, better packet delivery ratio, and less average delay. However, this method had similar weakness to previous method using minimum summation of the inverse SNR.

In summary, earlier methods that used SNR information as metric in channel-aware routing had two main schemes. First, the proposed scheme used routing discovery process, such as RREQ and RREP, to collect SNR information from the node. Second, to select the appropriate route, the earlier methods used average of SNR or summation of SNR of all the link. However, the use of SNR in earlier research, namely, by using the sum or the average of SNR, has a weakness because one or more links can have a very low SNR even though the average is high. This weakness will be explained further in Section 4. Furthermore, the use of SNR information in early researches on the route discovery process RREQ and RREP has increased overhead and complexity which should be avoided.

To overcome above problems, we propose an improved method which collaborates the cross-layers information, that is, the cooperation between the network and physical layer. In this scheme, we used same existing methods that employed SNR and cross-layer design to improve routing protocol performance. The most important aspects in our proposed methods, which have not been addressed in previous work and become our contributions, are as follows. Instead of adding SNR information to the RREQ or RREP process, we propose a selection of links for the reverse route mechanism based on SNR. This mechanism avoids additional overhead. Furthermore, selection route with a SNR threshold mechanism also improves the route choice compared to the previous minimum summation or average of the SNR methods. We additionally describe this crosslayer performance in network model and throughput formulation model based on SNR to give more explanation and analysis. 


\section{Network Model and Throughput Formulation}

3.1. Network Model. Since distributed network will influence its performance [6], in this subsection, we obtain and give explanation on the correlation between SNR of connection through propagation model and the expected number of hops as well as the average number of connections. Through this model, we can analyze the correlation between node density, node distribution, and the SNR connection. The performance of the network will be influenced by SNR connection, as described later in Section 3.2. For network model, we use an $N$-node network uniformly distributed over an area of a square with length of $2 d$. Based on [26, 27], we can calculate the expected number of hops and the average number of connections as follows. If the node density is $\delta=N /(2 d)^{2}$ and the node transmission range is $\tau$, then the number of average neighbors is $g=\left(N /(2 d)^{2}\right) \pi \tau^{2}-1$. The average distance between a node pair is given by $(d / 3)(\sqrt{2}+\ln (1+\sqrt{2}))$ and the average number of hops is $E_{\mathrm{hc}}=(d / 3 \tau)(\sqrt{2}+\ln (1+\sqrt{2}))$.

We can calculate the total number of network links as $g N / 2$. If we assume the number of connections is $Q$, then we approximate the average number of connections by $N_{\mathrm{cl}}=$ $2 Q k /(g+1) N$. For two-ray ground propagation model [28, 29], with the transmit power $\left(P_{t}\right)$, the system loss $(L)$, the separation distance between transmitter and receiver $\left(d_{s}\right)$, the gain transmit $\left(G_{t}\right)$, and the gain receiver $\left(G_{r}\right)$, thus we can calculate the power received in the receiver node given by $P_{r}(d)=P_{t} G_{t} G_{r} h_{t}{ }^{2} h_{r}{ }^{2} /\left(d_{s}\right)^{4} L$, with the height transmitter node $\left(h_{t}\right)$ and the height receiver $\left(h_{r}\right)$. If the SNR node is $\gamma$, with $G_{t}=G_{r}=L=1$, and $h_{r}=h_{t}=1.5$ meters from the ground, then we can calculate the expected number of hops per connection, $E_{\mathrm{hc}}$, and also the average number of connections over a given link, $N_{\mathrm{cl}}$, by

$$
\begin{aligned}
& E_{\mathrm{hc}}(\gamma)=\frac{2 d(\sqrt{2}+\ln (1+\sqrt{2})) \sqrt[4]{\gamma}}{9 \sqrt[4]{P_{t}}}, \\
& N_{\mathrm{cl}}(\gamma)=\frac{4 \mathrm{Qd}(\sqrt{2}+\ln (1+\sqrt{2})) \sqrt[4]{\gamma}}{9 N(g+1)\left(\sqrt[4]{P_{t}}\right)} .
\end{aligned}
$$

The SNR value of each connection will affect the expected number of hops per connection. The higher the SNR value, the better the ability of the network to have number of hops per connection. Figure 1 shows the SNR impact to expected number of hops per connection, with the difference of side length $(d)$, which are $250 \mathrm{~m}, 500 \mathrm{~m}$, and $1000 \mathrm{~m}$. We observed that increasing the side length of the network area also increased the expected number of hops per connection, especially in high SNR. The path connection consisting of links with high SNR has improved the ability of the network in obtaining better average number of connections, as shown in Figure 2 for fixed side length area. The links with high SNR provide a network to have better capability to create a path to deliver the data.

We observed that, with high SNR in the connection, the network could offer a big capability in terms of the average number of connections and the expected number

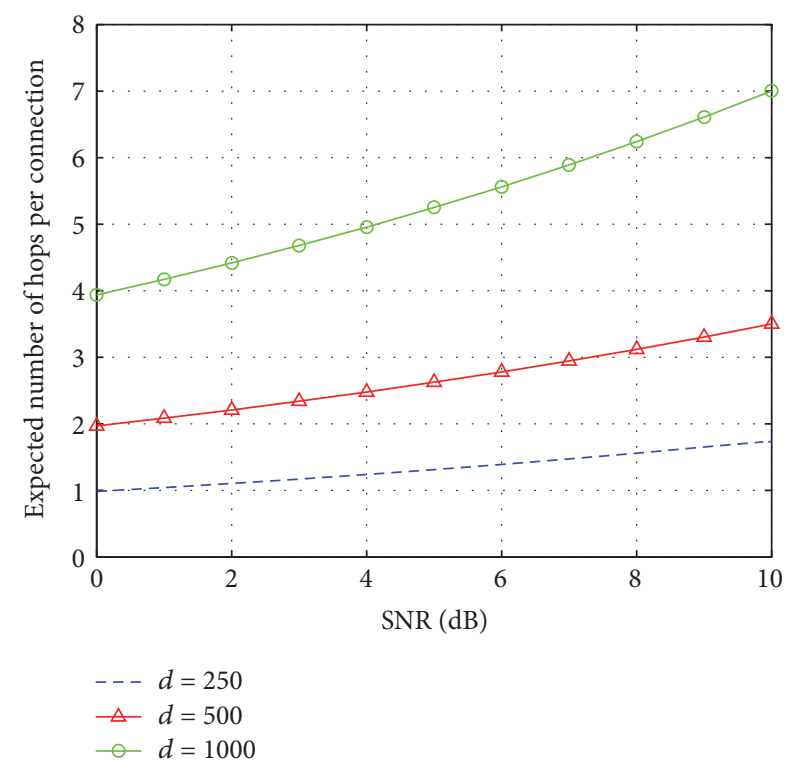

FIGURE 1: SNR effect on expected number of hops per connection with a different area length.

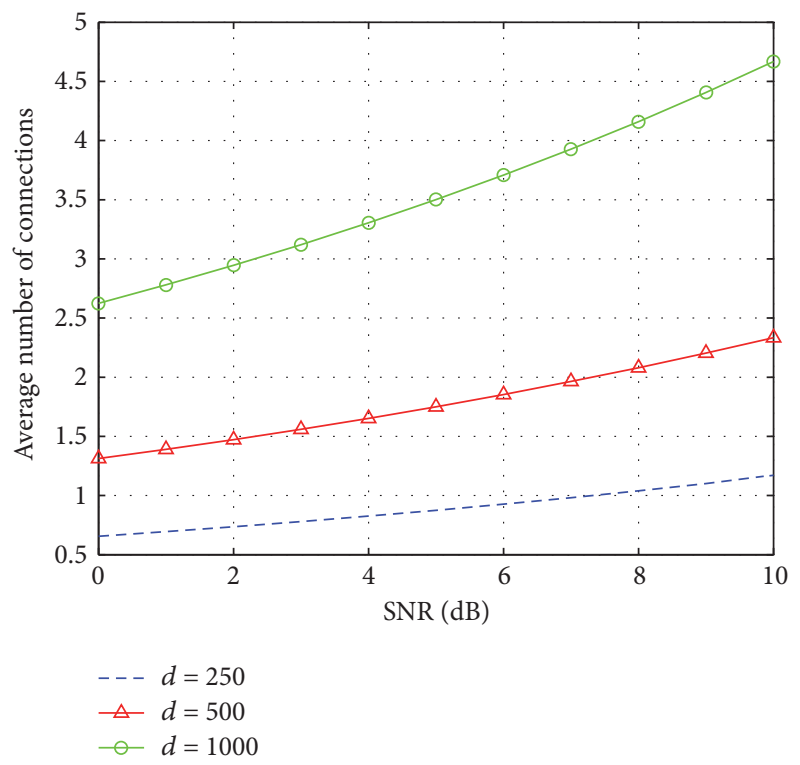

FIGURE 2: SNR effect on average number of connections with different area length.

of hops per connection, especially over wider area $(d)$. This capability can improve network performance. AODV SNRSR that considers SNR connection in the network will have opportunity to gain better average number of connections and the expected number of hops per connection to improve network performance.

3.2. Throughput Formulation. Throughput can be obtained by calculating the average number of successful data transmitted during the observation interval. Denote $B$ as the 
throughput, the average number of packets that successfully transmitted is $E[A]$, and the average duration of time required to transmit the data is $E[T]$; then

$$
B=\frac{E[A]}{E[T]} .
$$

If $R$ is average rate for delivery of the data and $\bar{K}$ is average of the data transferred, then the total average time to send $\bar{K}$ is $\bar{K} / R$ seconds. If $t_{p}$ represents the average propagation time and $t_{a}$ is the average time of the process at all nodes, then we can calculate $E[T]$ as

$$
E[T]=\frac{\bar{K}}{R}+2\left(t_{a}+t_{p}\right) .
$$

The quality of channel link and the mobility of node will have immediate effect on the probability of bit error. Let $P_{r}$ be the average probability of bits successfully delivered in the transmitted data. This probability of success is influenced by the probability of error in a connection and the number of hops. So we can rewrite $E[A]$ by

$$
E[A]=\bar{K} P_{r}
$$

while the throughput as function of $P_{r}$ and $R$ can be written as

$$
B=\frac{\bar{K} P_{r}}{\bar{K}+2 R\left(t_{a}+t_{p}\right)} R .
$$

If $p$ is the probability of error and $p_{i}$ is the probability of error on connection $i$, then the probability of success transmitting data on a connection $i$ is $\left(1-p_{i}\right)$. The total probability of success in sending the data for a path delivery that consists of $N$ links connection can be written as follows:

$$
P_{r}=\prod_{i=1}^{N}\left(1-p_{i}\right) .
$$

To analyze the correlation between the quality of the channel SNR with the throughput performance, we use the probability of error as bit error rate (BER) function [30], which is given by

$$
p=1-(1-\beta)^{n}
$$

where $\beta$ is the bit error rate and $n$ is the number of bits. We can calculate BER based on SNR and modulation technique. If Binary Phase Shift Keying (BPSK) modulation is used, the corresponding BER [31] is

$$
\mathrm{BER}=Q\left(\sqrt{2 \frac{E_{b}}{N_{o}}}\right),
$$

where $Q(\cdot)$ denotes the $Q$-function and $E_{b} / N_{o}$ denotes the energy per bit to the noise power ratio. If the signal bandwidth is approximately equal to the bandwidth of the noise or similarly equal to the receiver using a matched filter to get the maximum value of $S N R$, we can estimate $E_{b} /\left(N_{o} / 2\right) \approx S / N$.

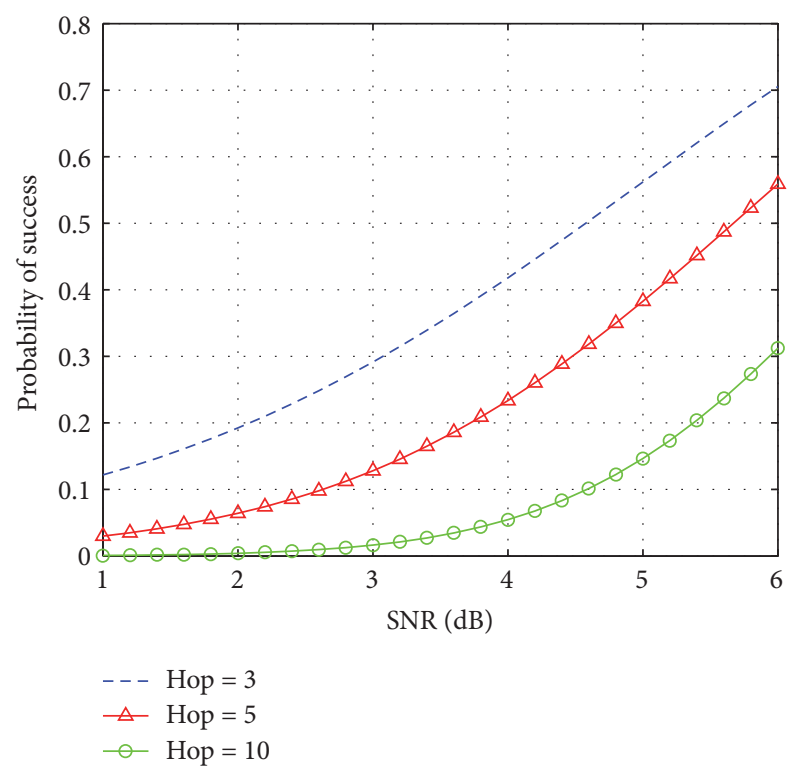

FIgURE 3: Probability of success with increasing SNR and different number of hops.

So, we can write the probability of error as a function of SNR as follows:

$$
p=1-\left(1-Q\left(\sqrt{\frac{S}{N}}\right)\right)^{n} .
$$

If we denote $\gamma=S / N$, then the throughput a function of $\gamma$ can be rewritten as

$$
B(\gamma)=\frac{\bar{K} \prod_{i=1}^{N}\left(1-Q(\sqrt{\gamma})_{i}\right)^{n}}{\bar{K}+2 R\left(t_{a}+t_{p}\right)} R .
$$

Equation (10) shows the throughput as a function of the probability of bit error as influenced by the SNR of the link connection. The probability of success to deliver the data will increase with higher SNR, as shown in Figure 3 with different number of hops scenario. To improve the throughput, the routing protocol should choose the link that has good SNR with a minimum probability of error. The simulation in Figure 4 shows that the throughput can gain higher performance by increasing SNR of the link connection with different transmission rate scenario. We observed that the probability of success and the throughput performance showed a significant improvement when the SNR connection increased.

Based on this fact and the mathematical model, we propose a selective algorithm with SNR as the parameter to choose the link as the path. To obtain a path that has a good SNR, the routing protocol must be well informed from the physical layer. For that purpose, we used cross-layer scheme as the method for routing protocol to get SNR information from the physical layer. We used SNR threshold $\left(\gamma_{\mathrm{tr}}\right)$ for the algorithm. A link can be selected by the routing protocol as a transmission line if $\gamma \geq \gamma_{\mathrm{tr}}$. With this algorithm, the routing protocol AODV SNR-SR will select link as the path, based on 


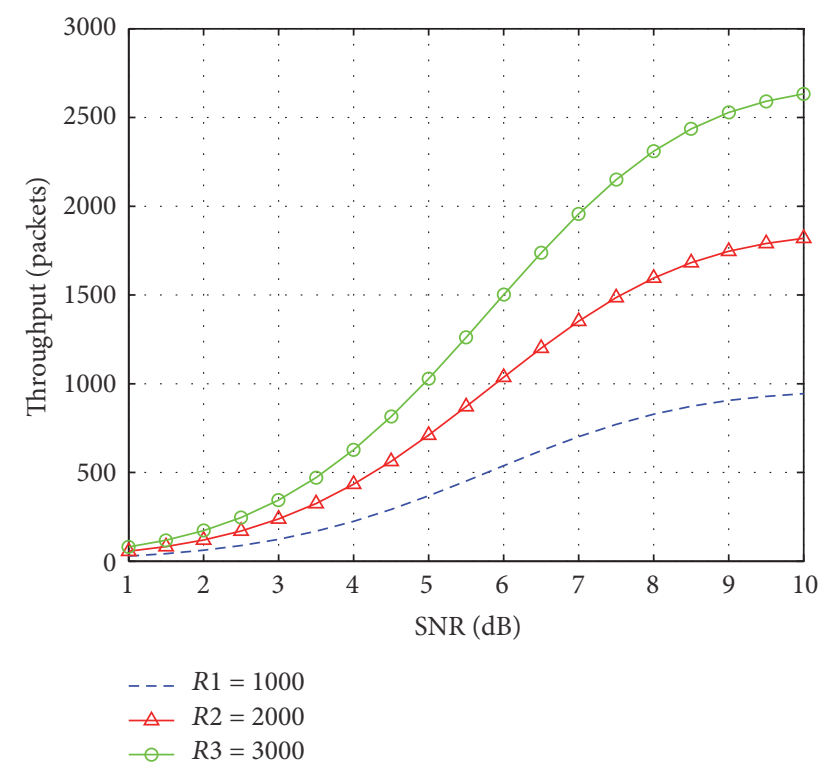

FIGURE 4: Throughput (packets) performance over different transmission rate (packets) with increasing SNR of link connection.

SNR values that meet the criteria. This routing algorithm can improve the network resilience against path break and low throughput by helping the network to find a reliable path base on SNR. The details of this method will be explained further in Section 4.

\section{SNR-Based Selective Reverse Route with Cross-Layer Scheme}

Generally, routing protocols select the path by minimizing the number of hops. However small number of hops usually corresponds to the selection of links with long transmission range and less capacity than the best paths that exist in the network. This long transmission range corresponds to low value of SNR. In ad hoc network, this poor SNR selection of link often produces the link-break, thus degrading overall network performance.

In previous researches, to get SNR information, the algorithm uses route discovery process, by adding the information in RREQ and RREP mechanism. Since RREQ mechanism broadcasts to all the neighbors and RREP unicasts, these methods increase the routing overhead and the complexity. Moreover, the algorithm used the minimum summation of the inverse SNR or the average of SNR value of the path. We call it AODV SNR.

This implementation could be inappropriate and cause the selected path to perform not as expected. It has weakness as one or more links can have a very low SNR even though the average is high. In this study, we use reactive routing protocol AODV [32] as the rule model, which is widely used. To show the importance of this issue, we illustrate this problem as shown in Figure 5.

The solid line represents the connection between nodes. The link channel quality is represented by SNR value, next

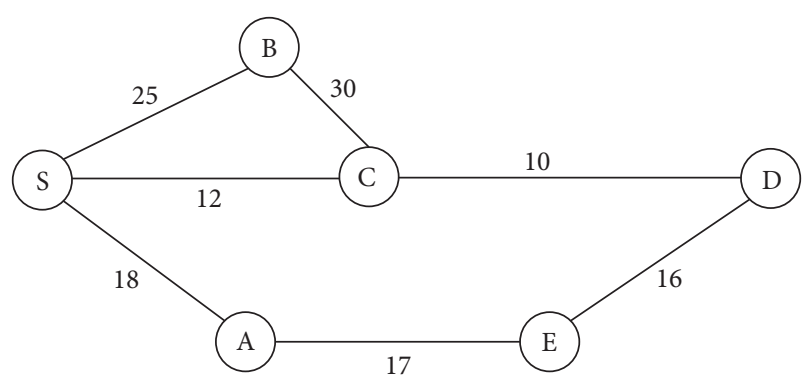

Figure 5: Illustration of network path.

to the node, with node $\mathrm{S}$ as the source and node $\mathrm{D}$ as the destination. For example, link S-A has SNR $=18 \mathrm{~dB}$ and link E-D has SNR $=16 \mathrm{~dB}$. These values are estimated at receiver nodes $A$ and $D$, respectively. In this case, to make a connection, a minimum SNR of $10 \mathrm{~dB}$ is required to get the maximum transmission range.

The routing protocol with hop count metric will choose the smallest number of hops as the path to deliver the data, which is S-C-D, with 2 hops. We can see that these hops consist of link that has low SNR to get the smallest hop. From (10), the throughput performance will decrease in connection with low SNR. Moreover, the mobility will cause this connection with low SNR to become vulnerable to the link-break, particularly if the nodes move to directions that decrease the SNR among them.

In AODV SNR, the routing protocol chooses the path that has the largest sum or largest average of the SNR. In this case, the AODV SNR will choose the path S-B-C-D, with the total of SNR being $65 \mathrm{~dB}$ or in the average $21.7 \mathrm{~dB}$. When we use minimum summation of inverse SNR, the routes' metric for routes (1) (S-B-C-D), (2) (S-C-D), (3) (S-A-E-D) is equal to $0.1733,0.1833$, and 0.1768 , respectively. Therefore, the routing with minimum of inverse SNR metric will select route 1 as the best route, same with the average SNR metric. Unfortunately, although it has an average of the largest SNR or the minimum summation of inverse SNR, the path may include link with low SNR, which is link C-D. The path with long trajectory with larger hops may have more links with very low SNR. In this situation, the link with low SNR will cause degradation of the throughput. Similar situation also occurs in hop count method, especially in mobility circumstances.

In this study, to solve the problem, we propose a new method, which is selective route based on SNR. We enhanced the reactive routing protocol AODV, and we call it as AODV SNR-SR. Link selection is done in the reverse route process, which will be used by the RREP. Figure 6 shows the proposed cross-layer design in AODV SNR-SR.

When the source needs to transmit the data, RREQ is generated. Reverse route is created when an intermediate node receives a RREQ. This reverse route is stored in the node for RREP path. After RREQ arrived at the destination, the RREP will be generated by the destination. Using reverse route, a destination node can send RREP to the source. Reverse route entry consists of source IP address, source sequence number, number of hops to the source node, and 


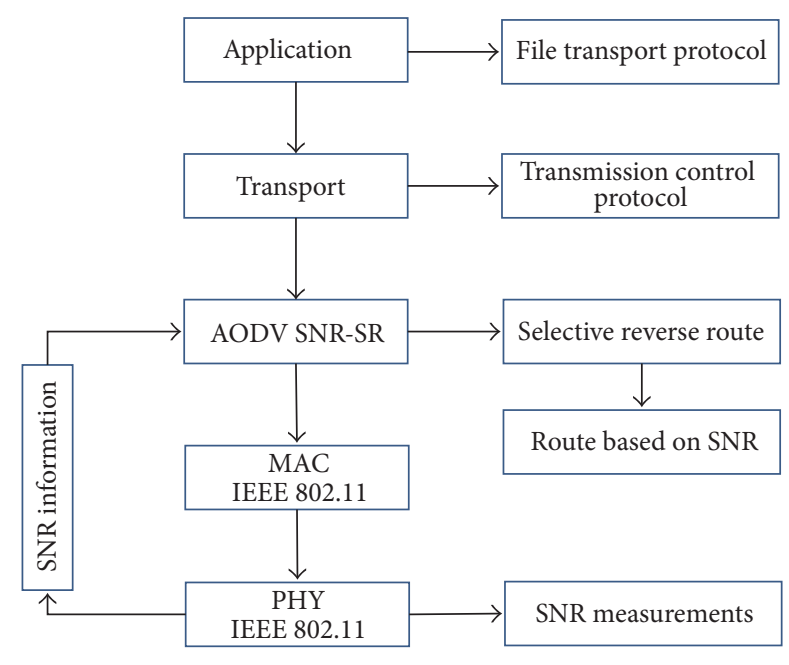

Figure 6: Cross-layer scheme AODV SNR-SR.

the IP address of the node from which RREQ is received. With this mechanism, when there is change on one of the links in reverse route, that is, path break, the RREP cannot be sent through this path and therefore uses another path. In cases where there is no available reverse route, then the source will generate new RREQ, after the time out. In AODV mechanism, source begins transmitting data when it receives the RREP, which has arrived first. The source will send the data to the destination using this reverse route.

With those mechanisms, it is important to select the appropriate reverse route based on SNR to get the best route. In AODV SNR-SR, selective route is conducted in the reverse route process. To avoid undesired small SNR selection in route path, we use minimum SNR threshold, which is $\gamma_{\mathrm{tr}}$. We define SNR threshold as the minimum requirement of the SNR received by a node, so that a given connection or path can be selected as a route by the routing algorithm. This SNR threshold needs to be set in all nodes. When a node receives a RREQ, it calculates the SNR $(\gamma)$ and compares it with SNR threshold; if the results meet the criteria $\gamma \geq \gamma_{\mathrm{tr}}$, the reverse route will be made and saved as route cache. When there is no available reverse routes that meet the criteria, the source will enter new discovery process.

If $\operatorname{SNR}(\gamma)$ criteria are not met, the reverse route is not created by the node, so the RREP cannot use this link. This filter makes RREP only use the reverse routes that meet the criteria; therefore, the sender only receives RREP with the route that has good SNR. In this case of AODV SNR$\mathrm{SR}$, the scheme will choose path S-A-E-D, where $\gamma_{\mathrm{tr}}=$ $15 \mathrm{~dB}$. SNR is a measure for determining the quality of a signal as it is disturbed by noise. The calculation can be done by determining the value obtained from the power of information signal and noise signal. The connection between nodes will have SNR value that depends on the mobility of both nodes. In this model, the SNR values are calculated with SNR $=10 \log \left(P_{\text {rss }} / N_{0}\right)$, where $P_{\text {rss }}$ is the received signal strength and it is assumed that the noise value is constant and same on all nodes. We used noise floor $\left(N_{o}\right) 2.512 e-$ 13 corresponding to thermal noise of the system [33]. In this study, we use two-ray ground propagation model to calculate the power at receiver, as described in network model section. The two-ray ground reflection model considers both the direct path and ground reflection path as multipath component. In our model, we assume that link qualities in both directions are identical. The reason is that, in ad hoc network, distribution of nodes within service area would have a direct path as well as a reflected path from the ground when the distance between nodes is short. In this propagation model, the forward and the reverse links would have the same characteristics in terms of path loss. In our model, nodes would have the same antenna height, located closely one to another, so we use two-ray ground propagation model.

The motivation of using SNR selection in reverse path mechanism is to avoid overhead, whereas other modifications on AODV generally use RREQ and RREP to gather SNR information, that would increase the size and complexity of the routing packet control. SNR threshold is used to avoid selection of one or more link with very low SNR as a part of the route. The mechanism of the route discovery process and implementation of selective reverse route algorithm in AODV SNR-SR can be seen in Figure 7. Route recovery mechanism works when there is a link-break at the transmission path, with Route Error (RERR) notification to the sender as depicted in Figure 8. When the intermediate node fails to deliver the data to the next hop due to path break and there is no other available paths, this node will generate RERR and send it to the source through the reverse path. After receiving this RERR, the source will create a new route discovery process to find a new path to transmit the data.

\section{AODV SNR-SR Performance and Discussion}

We evaluated the performance of AODV, AODV SNR, and AODV SNR-SR with Network Simulator (NS). The NS version for this particular simulation is version 2.35 [34]. In networks simulator, we enhanced the wireless-phy.cc to get power reception based on propagation model and then computed the SNR value. This SNR information is used by AODV SNR-SR to select appropriate route. We set the SNR threshold in route process by enhancing the aodv.cc. We varied the number of nodes and velocity in simulation scenario. We evaluated throughput, end-to-end delay, and routing overhead performance. The mobility model was random waypoint with simulation area of $1,000 \mathrm{~m} \times 1,000 \mathrm{~m}$. The data packet was 512 bytes, with channel bandwidth $2 \mathrm{Mb} / \mathrm{s}$, noise floor $\left(N_{o}\right) 2.512 e-13$, and simulation time 300 seconds. Maximum node speeds were $5 \mathrm{~m} / \mathrm{s}, 10 \mathrm{~m} / \mathrm{s}, 15 \mathrm{~m} / \mathrm{s}$, and $20 \mathrm{~m} / \mathrm{s}$ and numbers of nodes were 20,70,120, and 170 , respectively. Table 1 shows the simulation parameters. For single connection between two nodes, the correlation between the received SNR and the distance in two-ray ground propagation model is shown in Figure 9. The deployment of D2D in ad hoc network can use Wireless Fidelity (WIFI) with range up to more $100 \mathrm{~m}$ or WIFI direct as new technology with the range up to $200 \mathrm{~m}[35,36]$. Based on these facts, we found the distance that satisfies the D2D with WIFI direct technology and is adequate to set as the SNR threshold. We used SNR threshold $35 \mathrm{~dB}$ in the simulation, 


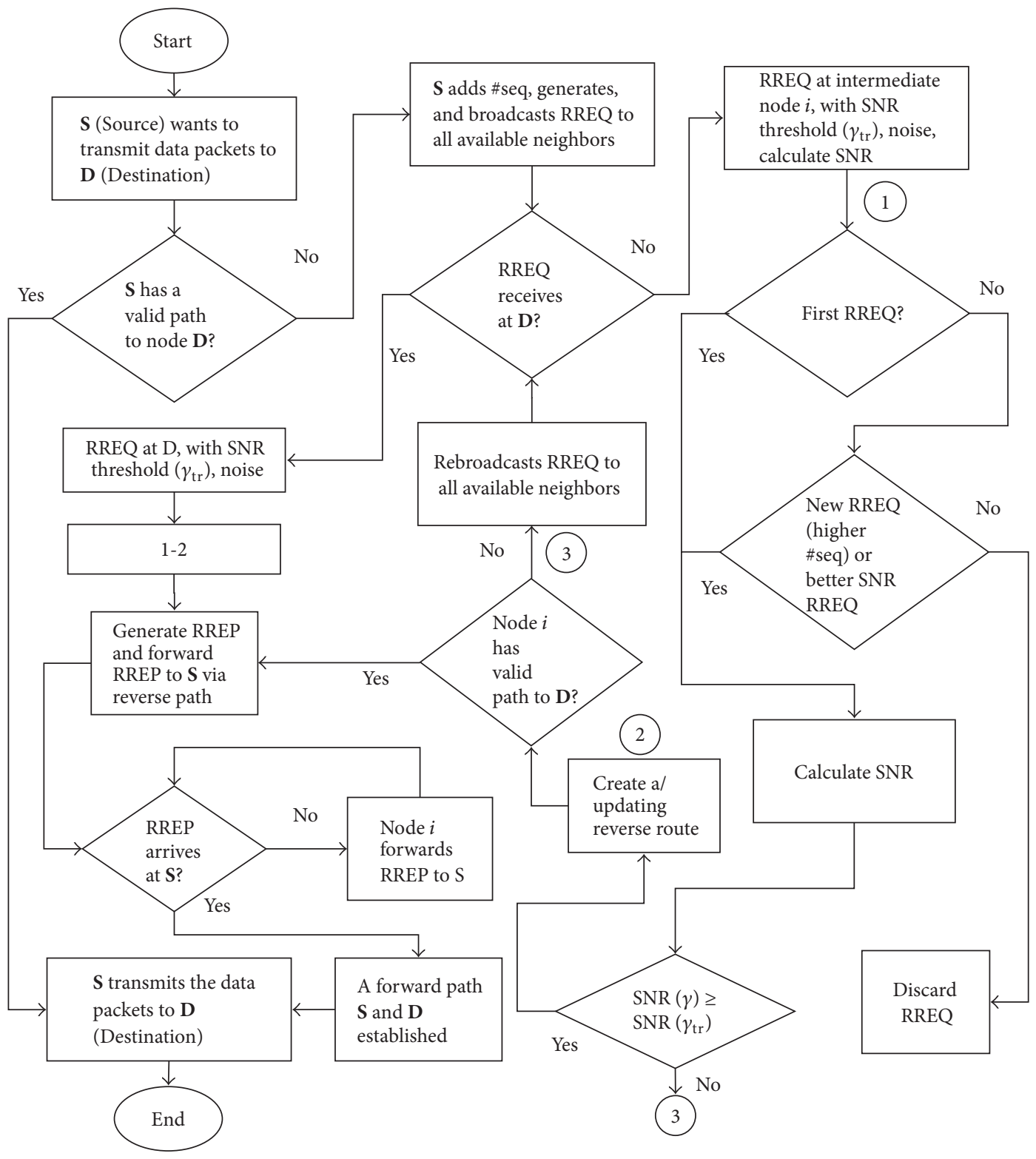

FIGURE 7: AODV SNR-SR route discovery process.

TABLE 1: Simulation parameters.

\begin{tabular}{lc}
\hline Mobility model & Random waypoint \\
Propagation model & Two-ray ground \\
Simulation duration & 300 seconds \\
Number of nodes & $20,70,120,170$ nodes \\
Simulation area & $1000 \mathrm{~m} \times 1000 \mathrm{~m}$ \\
Max node speed & $5 \mathrm{~m} / \mathrm{s}, 10 \mathrm{~m} / \mathrm{s}, 15 \mathrm{~m} / \mathrm{s}, 20 \mathrm{~m} / \mathrm{s}$ \\
Antenna & Omni antenna \\
Modulation & BPSK \\
\hline
\end{tabular}

within the distance range of $150 \mathrm{~m}$. This proposed method expected to support D2D communication in the environment that considers a ground-reflected propagation path between transmitter and receiver, in addition to the direct LOS (Line of Sight) path.
In Figure 10, we display the advantage of the proposed method compared to AODV and AODV SNR. In small number of nodes, the proposed method has a small advantage average throughput. However, the proposed method showed significant improvement of average throughput when the number of nodes increased. For example, in 170 nodes, the advantage of proposed method in average throughput was about $36 \%$ and $80 \%$ higher as compared to AODV SNR and AODV schemes. AODV SNR-SR also gained advantage when the number of nodes increased; the average end-to-end delays were about $30 \%$ and $20 \%$ smaller as compared to AODV and AODV SNR, respectively, as presented in Figure 11.

We analyzed the reasoning of results that appeared in Figures 10 and 11 as follows. The increasing number of nodes will correspondingly increase both the density of nodes and the average number of neighbors. If the area of side length is 


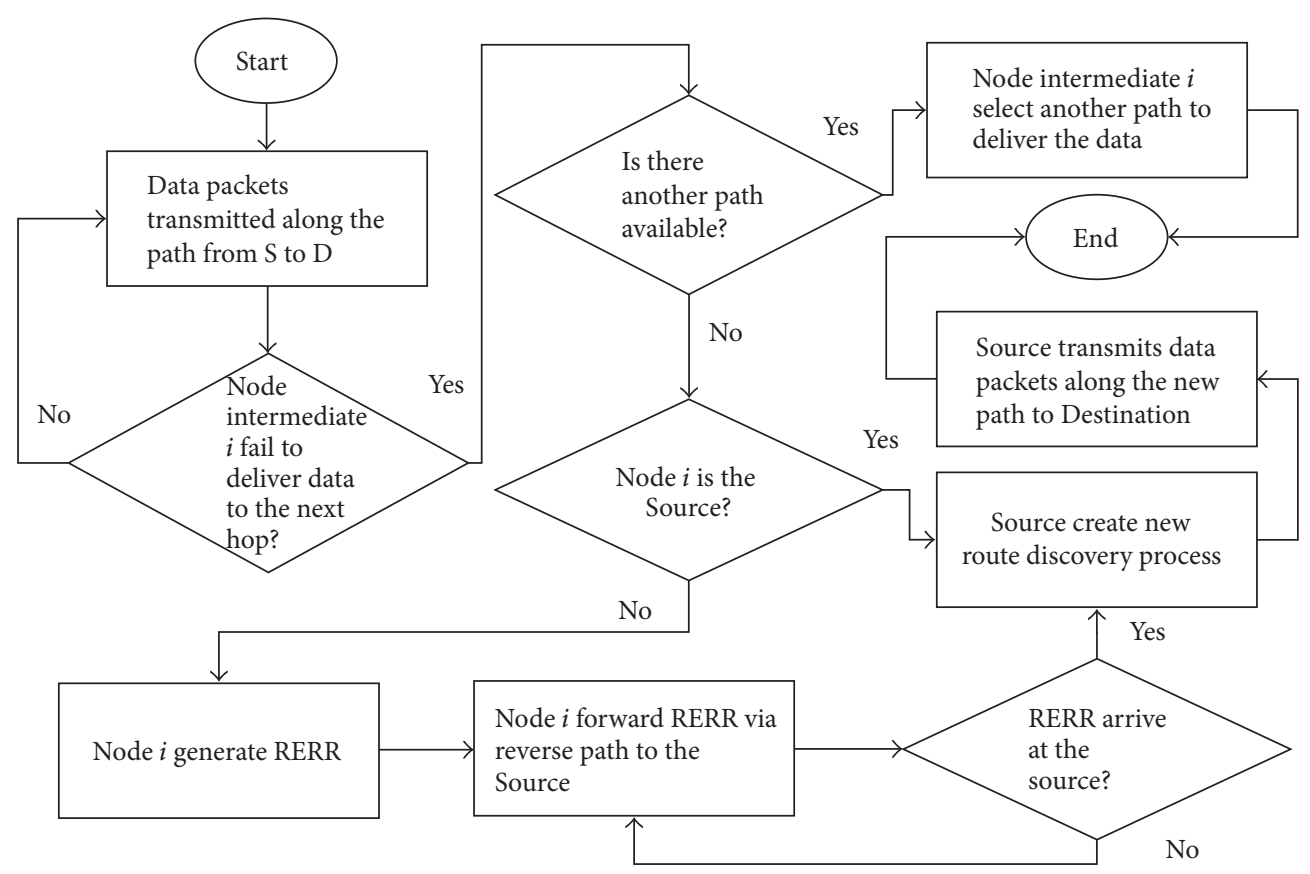

FIGURE 8: AODV SNR-SR route recovery.

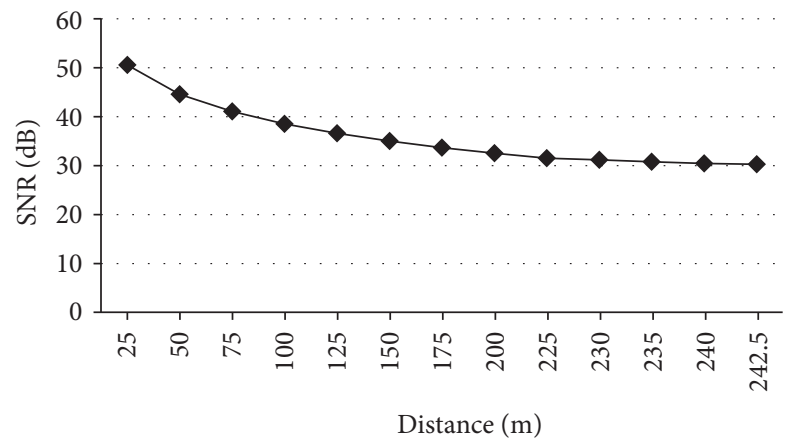

FIGURE 9: Received SNR in single connection with two-ray ground propagation model.

fixed, then such increasing number of nodes will also increase the total number of network links that can be created, as described in network models in Section 3.1. This situation makes the routing protocol easier to find possible routes, especially connection with high SNR. When the path consists of connections with high SNR, the throughput performance will increase as described in (10). The path with good quality connections will reduce the path breaks and allow more stability in sending the data, therefore decreasing the average end-to-end delay.

Simulation results for routing overhead per received data packet are shown in Figure 12, where AODV SNR-SR outperformed AODV SNR and AODV, with smallest routing overhead. We observed that, in small number of nodes, routing protocol produced small overhead; however, with the increasing numbers of nodes, the overhead rapidly became larger. The proposed method showed a big advantage which

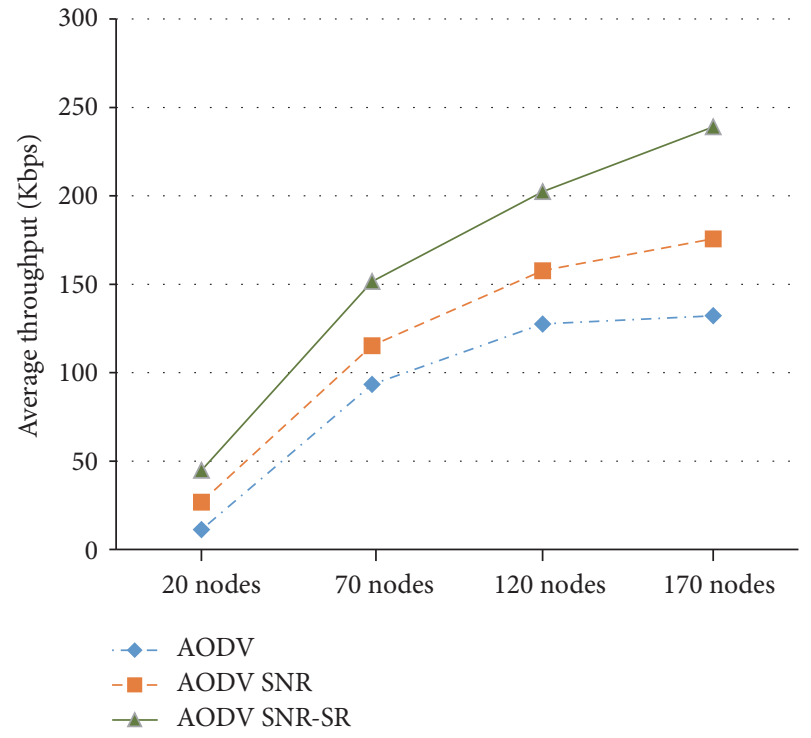

FIgURE 10: Average throughput comparison between AODV, AODV SNR, and AODV SNR-SR with increasing number of nodes.

was about $59 \%$ and $36 \%$ smaller overheads as compared to AODV and AODV SNR.

We also compared AODV SNR-SR, AODV SNR, and AODV with respect to node velocity. The AODV SNR-SR outperformed the AODV SNR and AODV in terms of smaller overhead per received data packet in node velocity scenario as shown in Figure 13. We observed that the proposed scheme was about $44 \%$ and $59 \%$ more efficient in routing overheads compared to AODV SNR and AODV, respectively. These results show good agreement with (14), where routing 


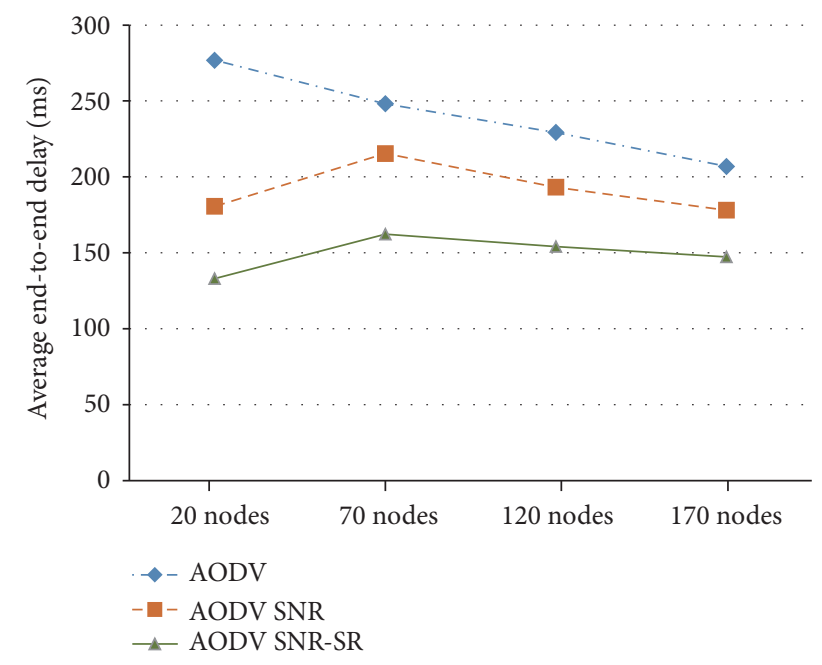

FIGURE 11: Average end-to-end delay comparison between AODV SNR-SR, AODV SNR, and AODV with increasing number of nodes.

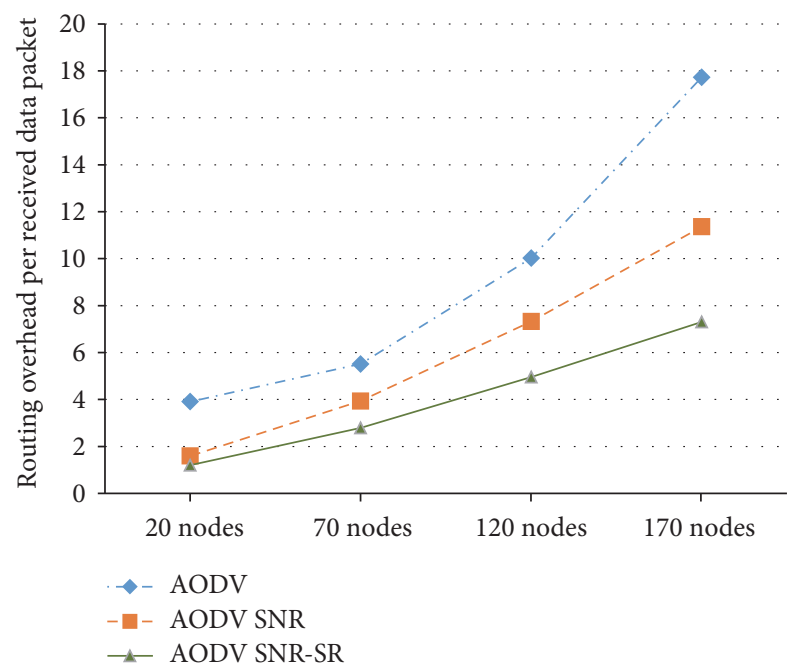

FIGURE 12: Routing overhead (normalized) comparison between AODV, AODV SNR, and AODV SNR-SR with increasing number of nodes.

overhead will have immediate effect on the network efficiency based on SNR. The analysis and the reasoning of the results in Figures 12 and 13 can be described as follows. Normalized routing overhead is calculated as the ratio of the total routing control packets to deliver data packets. Routing control packets included RREQ, RREP, and a RERR. This RERR is triggered by link-break to give notification to the sender. If the sender does not have alternative route, it will broadcast RREQ again and enter the route discovery process.

The routing overhead corresponding to network performance can be analyzed as follows. We can evaluate the efficiency of the network $(\eta)$ for a single link by calculating the average total number of bits being transferred during time $T$, which is $\alpha$. The total bits to process forward route RREQ are $\beta_{\text {RREQ }}$.

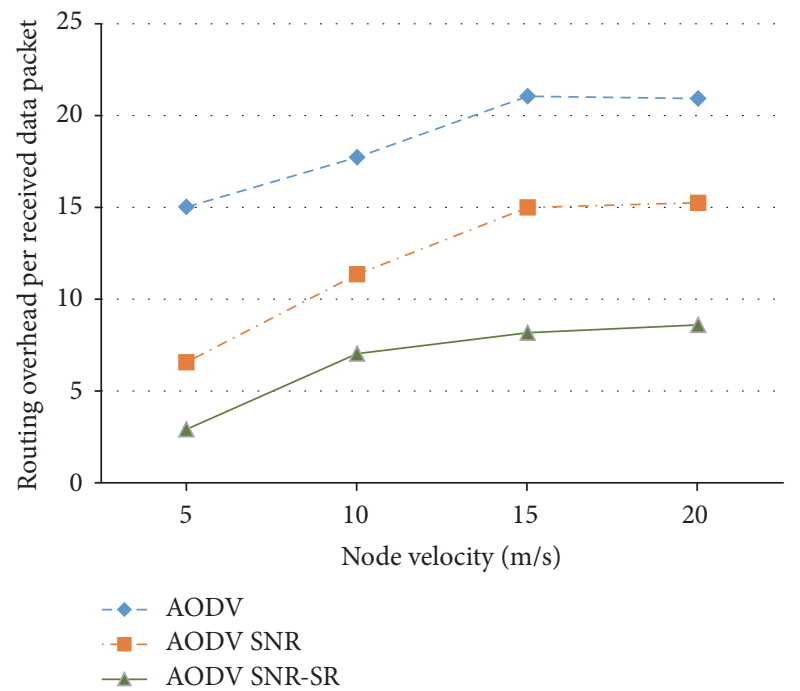

FIGURE 13: Routing overhead (normalized) comparison between AODV, AODV SNR, and AODV SNR-SR with increasing node velocity.

Denote $\varphi_{\text {RREP }}$ to be the total number of bits as the acknowledgment route process RREP and $\varepsilon_{\text {RERR }}$ to be the total bits notification of route failure, RERR. If the effective rate transmission is $R_{\text {eff }}$ and transmission rate is $R$, then we can express the efficiency as $\eta=R_{\text {eff }} / R$. If the probability of error $p$ for $x$ transmission can be expressed as

$$
p(x)=p^{x-1}(1-p),
$$

then the mean of this probability of error $\psi$ can be expressed as

$$
\psi=\sum_{X=1}^{\infty} x p^{x-1}(1-p)=\frac{1}{1-p}
$$

If we use a model approach in selective ARQ [30], then we can find the efficiency of the network-based routing overhead which is related to probability of error by

$$
\eta=\frac{\alpha-\beta_{\mathrm{RREQ}}}{(1 /(1-p))\left(\alpha+\varphi_{\mathrm{RREP}}+\varepsilon_{\mathrm{RERR}}\right)},
$$

and as an SNR function we derive efficiency as

$$
\eta(\gamma)=\frac{\left(\alpha-\beta_{\mathrm{RREQ}}\right)\left(1-Q(\sqrt{\gamma})_{i}\right)^{n}}{\left(\alpha+\varphi_{\mathrm{RREP}}+\varepsilon_{\mathrm{RERR}}\right)} .
$$

We can conclude from (13) that the increasing routing overhead will reduce network efficiency and throughput. From (14), the route with link that has better SNR is also more efficient. SNR on link connection will have an impact on the probability of error and link failure. This will influence the amount of RERR and the path break will trigger RERR. Accordingly, if the source does not have any routes, it will start route discovery process and generate RREQ. This mechanism will increase the routing overhead. 


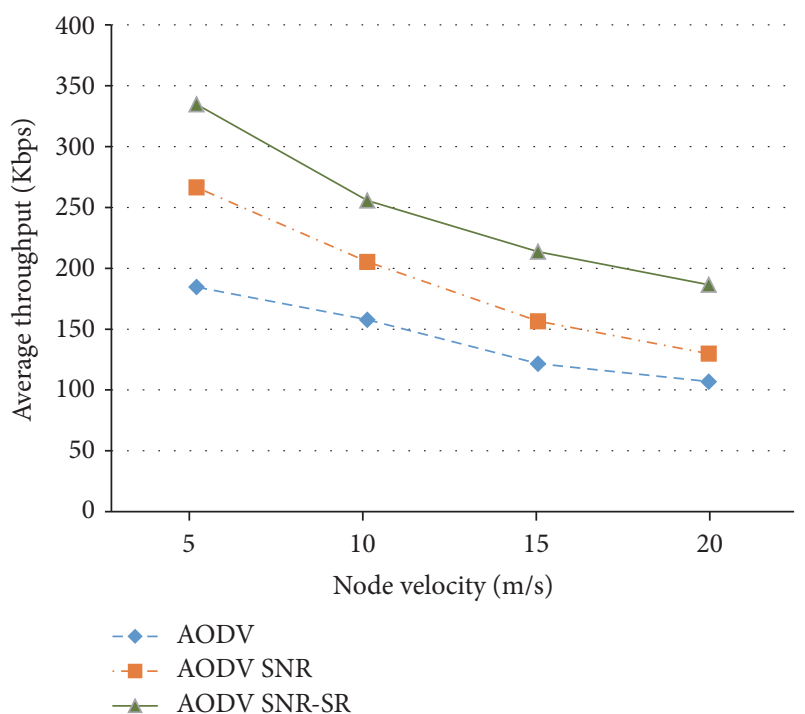

FIGURE 14: Average throughput comparison between AODV, AODV SNR, and AODV SNR-SR with increasing node velocity.

Simulation results for the throughput are shown in Figure 14 with increasing node velocity. The throughput decreased along the increase of node velocity, with AODV SNR-SR outperforming AODV SNR and AODV. We observed that the proposed method yielded significant improvement of throughput when the velocity increased, in average about $45 \%$ and $78 \%$ higher compared to AODV SNR and AODV.

The simulation results for the end-to-end delay with respect to node velocity are shown in Figure 15. The proposed AODV SNR-SR again outperformed AODV SNR and AODV. We observed that, in slow velocity, the end-to-end delay performance varied less between AODV SNR and AODV SNR-SR. However, in high velocity, the AODV SNR-SR got advantages with smaller end-to-end delay about $31 \%$ and $26 \%$ compared to AODV and AODV SNR.

From the results in Figures 14 and 15, we analyzed the following. When the node velocity increases, the distribution of the network becomes more dynamic. This situation leads the SNR connection to be more unstable, and often path break occurs. Consequently, the throughput will decrease and endto-end delay becomes higher. To analyze correlation between end-to-end delay and the SNR connection in Figures 11 and 15 , we can use (3), (9), and (12). For single connection with the average probability of error $\psi$ for $x$ transmission, we calculate the end-to-end delay $\left(D_{\mathrm{e} 2 \mathrm{e}}\right)$ for one-way delay by

$$
D_{\mathrm{e} 2 \mathrm{e}}=\frac{\bar{K}+R\left(t_{a}+t_{p}\right)}{R\left(1-Q(\sqrt{\gamma})_{i}\right)^{n}}
$$

From (15), we can see that higher SNR connection will produce smaller end-to-end delay; this is the reason why network performance increases. AODV SNR-SR is capable of maintaining performance due to higher SNR connection in the route. We analyzed the correlation between the velocity of nodes and the network performance as follows. The

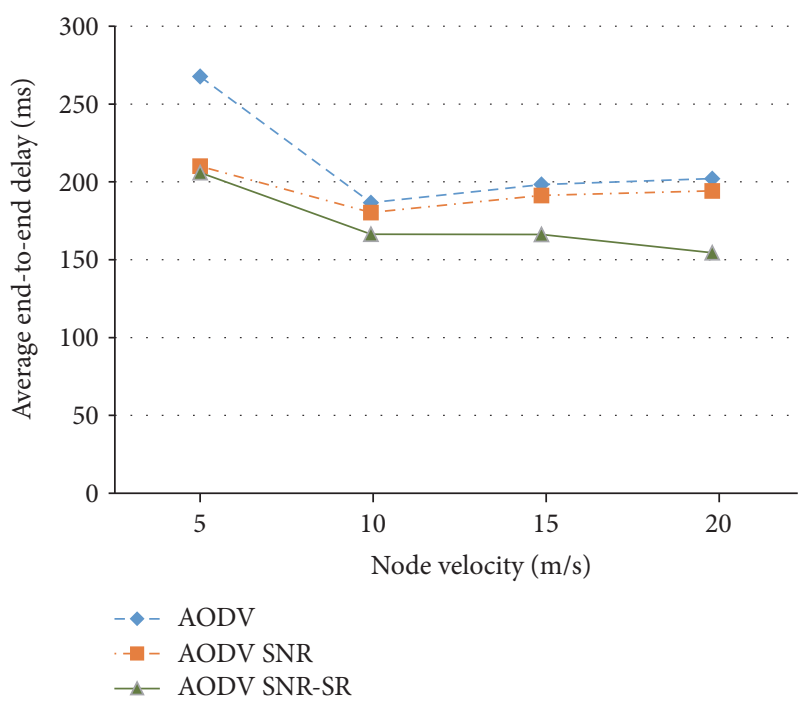

FIGURE 15: Average end-to-end delay comparison between AODV SNR-SR, AODV SNR, and AODV with increasing node velocity.

average duration of the path connection $(\rho)$ depends on the transmission range $(R)$, number of hops $(s)$, and the velocity of the node $(V)$. If we use mobility models which are exponentially distributed with parameter $\sigma$ for the average duration of the path connection [37], then we can get

$$
\rho=\frac{1}{\sigma}=\frac{1}{\sigma_{0}} \frac{R}{s V}
$$

and the probability density function is given by

$$
f(x)=\frac{\sigma_{0} s V}{R} e^{-\left(\sigma_{0} s V / R\right) x},
$$

where $\sigma_{0}$ is the constant of proportionality and is independent from $R, s$, and $V$. From (16) and (17), we can see that velocity of node will decrease the average duration of connection path and induce the degradation of network performance. The higher average of connection path with better SNR connection is, the lower end-to-end delay and improved throughput will be produced. Transmission range will depend on both the distribution and the density of nodes as described in Section 3, that SNR of connection will have direct effect on the throughput performance. The reasoning of velocity effect on routing overhead with results shown in Figure 13 is described as follows. From (16), we also can approximate the routing overhead that is influenced by the velocity of the node, which is $T_{s} / \rho$, where $T_{s}$ is time simulation. When the velocity increases, it will result in lower average duration of the path connection and higher routing overhead.

In this proposed mechanism, the performance will depend on the network model as mentioned in Section 3.1 and the SNR threshold. The density and distribution of the network will have an impact on the SNR connection. Since the distribution and the mobility of the node in network are difficult to predict, there is situation where SNR connection cannot meet the SNR threshold. This situation makes the 


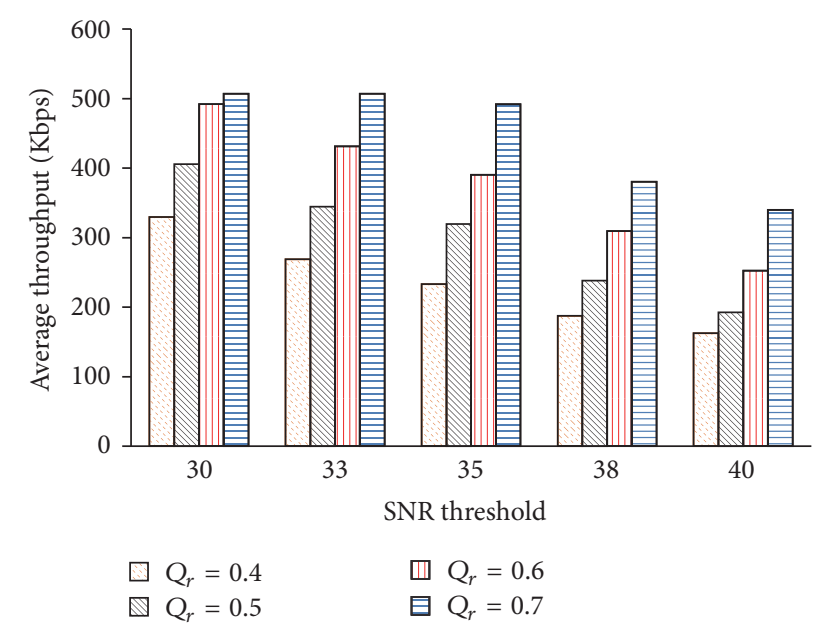

FIgURE 16: Average throughput as minimum and maximum SNR ratio $\left(Q_{r}\right)$ with different SNR threshold.

routing protocols unable to find the suitable routes that agree with channel quality requirement. Another situation is the node fail to deliver the data to the next hop due to the path break or there are no available paths. When this situation happens, a new route discovery process must be undertaken.

We analyzed the impact of the SNR threshold and the distribution of SNR connection to the network performance as follows. Figure 16 shows the correlation between SNR threshold, the $Q_{r}$ factor, and the average throughput performance. The $Q_{r}$ factor is defined as the ratio of minimum SNR compared to maximum SNR on the distribution of the SNR connections, which is $Q_{r}=\mathrm{SNR}_{\min } / \mathrm{SNR}_{\max }$. We set maximum SNR connection to $50 \mathrm{~dB}$ and minimum SNR connection to $20 \mathrm{~dB}, 25 \mathrm{~dB}, 30 \mathrm{~dB}$, and $35 \mathrm{~dB}$. For the distribution of SNR connection between $20 \mathrm{~dB}\left(\mathrm{SNR}_{\text {min }}\right)$ and $50 \mathrm{~dB}\left(\mathrm{SNR}_{\max }\right)$, we get $Q_{r}=0.4$. We generated 100 random SNR connections using five different SNR thresholds, which were $30 \mathrm{~dB}, 33 \mathrm{~dB}, 35 \mathrm{~dB}, 38 \mathrm{~dB}$, and $40 \mathrm{~dB}$. In this scenario, the SNR connection that complies with the SNR threshold was assigned as route and the throughput of this connection are calculated. We observed that higher $Q_{r}$ factor improved the throughput of the network. When $\mathrm{SNR}_{\min }$ has wider gap to the SNR threshold, it will reduce the performance. On the other hand, it will increase the performance when $\mathrm{SNR}_{\text {min }}$ is close to the SNR threshold. When the SNR threshold is close to $\mathrm{SNR}_{\max }$, the increasing $Q_{r}$ factor will not greatly influence the throughput performance, also causing the routing protocol hard to find suitable path that meets the requirement. Although lower SNR thresholds seem to gain higher throughput, they can produce connection with lower SNR and cause degradation of network performance, especially in lower $Q_{r}$ factor. For example, SNR threshold $30 \mathrm{~dB}$ with $Q_{r}=0.4$ will produce throughput smaller than the SNR threshold $33 \mathrm{~dB}$ with $Q_{r}=0.5$.

In summary, AODV SNR-SR outperforms AODV SNR and the conventional AODV, because it gains more throughputs with better algorithm mechanism as described in Sections 3 and 4, respectively. The proposed scheme can also improve the route discovery efficiency and make routing protocol more adaptive with channel-aware for $\mathrm{D} 2 \mathrm{D}$ requirement as mentioned in $[6,8-10]$, where the conventional schemes cannot solve.

\section{Conclusion and Future Research Opportunities}

In this study, we developed throughput formulation and network model. We proposed new algorithm in routing protocol to increase the network performance. We proposed this algorithm by enhancing the AODV routing protocols with a cross-layer scheme based on SNR, termed as AODV SNR-SR. This proposed scheme employed selective route based on the SNR threshold in the reverse route process. These mechanisms avoid high routing overheads and low SNR selections in the route path. With these techniques, AODV SNR-SR outperforms AODV SNR and AODV in terms of throughput, end-to-end delay, and routing overhead. The AODV SNR-SR shows a significant advantage in throughput, end-to-end delay, and routing overhead when the number and the velocity of nodes increase. This algorithm can improve network resilience against path break and high throughput performance to support D2D development. For future work, SNR threshold mechanism has to be considered along with the application and the type of the data, especially for adaptive data rate.

\section{Conflicts of Interest}

The authors declare that there are no conflicts of interest regarding the publication of this paper.

\section{Acknowledgments}

This work is supported by Ministry of Research Technology and Higher Education of the Republic of Indonesia, Telkom University, and Yayasan Pendidikan Telkom.

\section{References}

[1] M. Riaz, N. M. Khan, and S. J. Nawaz, "A generalized 3-d scattering channel model for spatiotemporal statistics in mobileto-mobile communication environment," IEEE Transactions on Vehicular Technology, vol. 64, no. 10, pp. 4399-4410, 2015.

[2] A. Attwood, M. Merabti, and O. Abuelmaatti, "IoMANETs: mobility architecture for wireless M2M networks," in Proceedings of the IEEE GLOBECOM Workshops, GC (Wkshps '11), pp. 399-404, Houston, TX, USA, December 2011.

[3] S. Mumtaz, K. M. Saidul Huq, and J. Rodriguez, "Direct mobileto-mobile communication: paradigm for 5G," IEEE Wireless Communications, vol. 21, no. 5, pp. 14-23, 2014.

[4] S. Riaz, H. K. Qureshi, and M. Saleem, "Performance evaluation of routing protocols in energy harvesting D2D network," in Proceedings of the 1st International Conference on Computing, Electronic and Electrical Engineering, (ICE Cube '16), pp. 251255, Quetta, Pakistan, April 2016.

[5] B. Kaufman, J. Lilleberg, and B. Aazhang, "Spectrum sharing scheme between cellular users and ad-hoc device-to-device users," IEEE Transactions on Wireless Communications, vol. 12, no. 3, pp. 1038-1049, 2013. 
[6] Y. Wu, S. Wang, W. Liu, W. Guo, and X. Chu, "Iunius: a crosslayer peer-to-peer system with device-to-device communications," IEEE Transactions on Wireless Communications, vol. 15, no. 10, pp. 7005-7017, 2016.

[7] R. Edirisinghe and A. Zaslavsky, "Cross-layer contextual interactions in wireless networks," IEEE Communications Surveys \& Tutorials, vol. 16, no. 2, pp. 1114-1134, 2014.

[8] O. Bello and S. Zeadally, "Intelligent device-to-device communication in the internet of things," IEEE Systems Journal, vol. 10, no. 3, pp. 1172-1182, 2016.

[9] A. L. Beylot, R. Dhaou, V. Gauthier, and M. Becker, "Cross-layer simulation and optimization for mobile ad-hoc networks," in Mobile and Wireless Communication Networks, E. M. BeldingRoyer, K. Al Agha, and G. Pujolle, Eds., vol. 162 of IFIP International Federation for Information Processing, pp. 13-22, Springer, Boston, Mass, USA, 2005.

[10] J. Zuo, C. Dong, S. X. Ng, L. L. Yang, and L. Hanzo, "Cross-layer aided energy-efficient routing design for ad hoc networks," IEEE Communications Surveys \& Tutorials, vol. 17, no. 3, pp. 12141238, 2015.

[11] A. Zhou, M. Liu, Z. Li, and E. Dutkiewicz, "Joint traffic splitting, rate control, routing, and scheduling algorithm for maximizing network utility in wireless mesh networks," IEEE Transactions on Vehicular Technology, vol. 65, no. 4, pp. 2688-2702, 2016.

[12] K. Kunavut, "Link quality aware routing based on effective estimated throughput for mobile ad hoc networks," in Proceedings of the 21st International Symposium on Intelligent Signal Processing and Communication Systems, (ISPACS '13), pp. 487492, Naha, Japan, November 2013.

[13] D. De Couto, D. Aguayo, J. Bicket, and R. Morris, "A highthroughput path metric for multi-hop wireless routing," in Proceedings of the 9th Annual International Conference on Mobile Computing and Networking (MobiCom '03), pp. 134-146, San Diego, Calif, USA, September 2003.

[14] B. Sen, J. Guo, X. Zhao, and S. Jha, "ECTX: a high-throughput path metric for multi-hop wireless routing exploiting MAClayer cooperative retransmission," in Proceedings of the 13th IEEE International Symposium on a World of Wireless, Mobile and Multimedia Networks, (WoWMoM '12), pp. 1-9, San Francisco, CA, USA, June 2012.

[15] R. Draves, J. Padhye, and B. Zill, "Routing in multi-radio, multihop wireless mesh networks," in Proceedings of the 10th Annual International Conference on Mobile Computing and Networking (MobiCom '04), pp. 114-128, September 2004.

[16] B. Awerbuch, D. Holmer, and H. Rubens, "The medium time metric: high throughput route selection in multi-rate ad hoc wireless networks," Mobile Networks and Applications, vol. 11, no. 2, pp. 253-266, 2006.

[17] R. Agüero, J. A. Galache, and L. Muñoz, "Using SNR to improve multi-hop routing," in Proceedings of the IEEE 69th Vehicular Technology Conference (VTC Spring '09), pp. 1-5, Barcelona, Spain, April 2009.

[18] F. Alnajjar, "SNR/RP aware routing model for MANETs," Cyber Journals: Multidisciplinary Journals in Science and Technology, Journal of Selected Areas in Telecommunications, pp. 40-48, 2011.

[19] M. K. Islam and R. K. Liu, "Cross-layer optimization of AODV routing protocol for mobile ad-hoc network (MANET)," in Proceedings of the 2nd International Conference on Computer Science and Electronics Engineering (ICCSEE '13), pp. 1834-1837, 2013.
[20] B. Amiri, H. R. Sadjadpour, and J. Garcia-Luna-Aceves, "Outage optimum routing for wireless networks," in Proceedings of the 7th International Wireless Communications and Mobile Computing Conference, (IWCMC '11), pp. 1-6, Istanbul, Turkey, July 2011.

[21] M. Elshaikh, M. F. M. Fadzil, N. Kamel, and C. M. N. C. Isa, "Weighted signal-to-noise ratio average routing metric for dynamic sequence distance vector routing protocol in mobile ad-hoc networks," in Proceedings of the IEEE 8th International Colloquium on Signal Processing and Its Applications, (CSPA '12), pp. 329-334, Melaka, Malaysia, March 2012.

[22] S. Ganesh and R. Amutha, "Efficient and secure routing protocol for wireless sensor networks through SNR based dynamic clustering mechanisms," Journal of Communications and Networks, vol. 15, no. 4, pp. 422-429, 2013.

[23] A. M. Akhtar, A. Behnad, and X. Wang, "Cooperative ARQbased energy-efficient routing in multihop wireless networks," IEEE Transactions on Vehicular Technology, vol. 64, no. 11, pp. 5187-5197, 2015.

[24] Y. B. Nechaev and A. V. Stromov, "Interference aware routing in massively dense wireless networks based on streamlines of potential field," in Proceedings of the 39th International Conference on Telecommunications and Signal Processing (TSP '16), pp. 79-82, Vienna, Austria, 2016.

[25] A. Ben Ammar, A. Dziri, M. Terre, and H. Youssef, "Multihop LEACH based cross-layer design for large scale wireless sensor networks," in Proceedings of the International Wireless Communications and Mobile Computing Conference (IWCMC '16), pp. 763-768, Paphos, Cyprus, 2016.

[26] X. Chen, H. M. Jones, and D. Jayalath, "Channel-aware routing in MANETs with route handoff," IEEE Transactions on Mobile Computing, vol. 10, no. 1, pp. 108-121, 2011.

[27] S. Panichpapiboon, G. Ferrari, and O. K. Tonguz, "Optimal transmit power in wireless sensor networks," IEEE Transactions on Mobile Computing, vol. 5, no. 10, pp. 1432-1447, 2006.

[28] A. Goldsmith, Wireless Communications, Cambridge University Press, 2005.

[29] T. Rappaport, Wireless Communications: Principles and Practice, Prentice Hall, 2001.

[30] J. D. Spragins, J. L. Hammaond, and K. Pawlikowski, Telecommunications: Protocols and Design, Addison-Wesley Publishing, 1991.

[31] M. Khosroshahy, Study and Implementation of IEEE 802.11 Physical Channel Model in YANS (NS3 prototype) Network Simulator, INRIA-Sophia Antipolis-Planète Group, 2006.

[32] C. E. Perkins and E. M. Royer, "Ad-hoc on-demand distance vector routing," in Proceedings of the 2nd IEEE Workshop on Mobile Computing Systems and Applications (WMCSA '99), pp. 90-100, New Orleans, La, USA, February 1999.

[33] J. Kim and J.-M. Ahn, "Aggressive spatial reuse scheme for the 802.11 wireless LAN," The Journal of Korean Institute of Communications and Information Sciences, vol. 41, no. 2, pp. 222-228, 2016.

[34] Network Simulator, NS 2.35, http://www.isi.edu/nsnam/ns/ ns-build.html.

[35] A. Pyattaev, K. Johnsson, S. Andreev, and Y. Koucheryavy, "3GPP LTE traffic offloading onto WiFi Direct," in Proceedings of the IEEE Wireless Communications and Networking Conference Workshops (WCNCW '13), pp. 135-140, Shanghai, China, April 2013. 
[36] Wi-Fi Alliance. WIFI Direct, http://www.wi-fi.org/discover-wifi/wi-fi-direct.

[37] F. Bai, N. Sadagopan, B. Krishnamachari, and A. Helmy, "Modeling path duration distributions in MANETs and their impact on reactive routing protocols," IEEE Journal on Selected Areas in Communications, vol. 22, no. 7, pp. 1357-1373, 2004. 


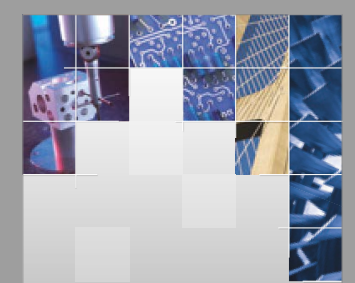

\section{Enfincering}
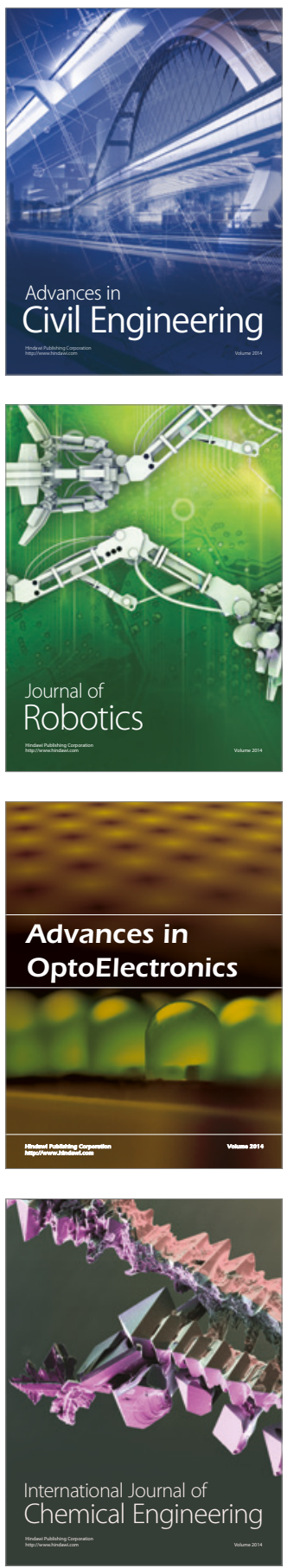

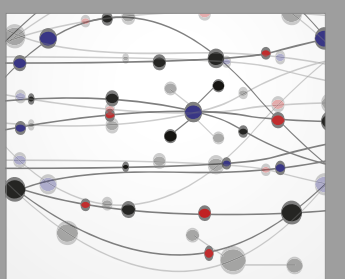

The Scientific World Journal

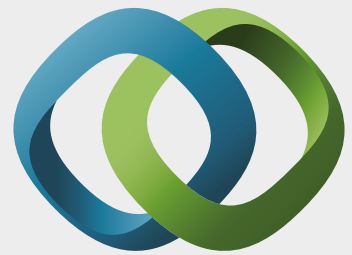

\section{Hindawi}

Submit your manuscripts at

https://www.hindawi.com
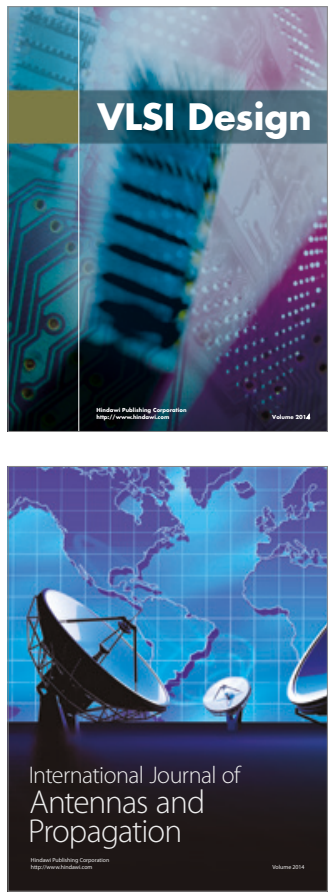

\section{Rotating}

Machinery
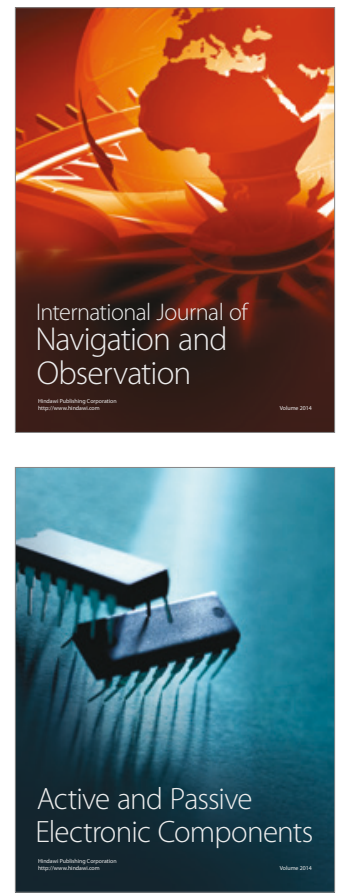
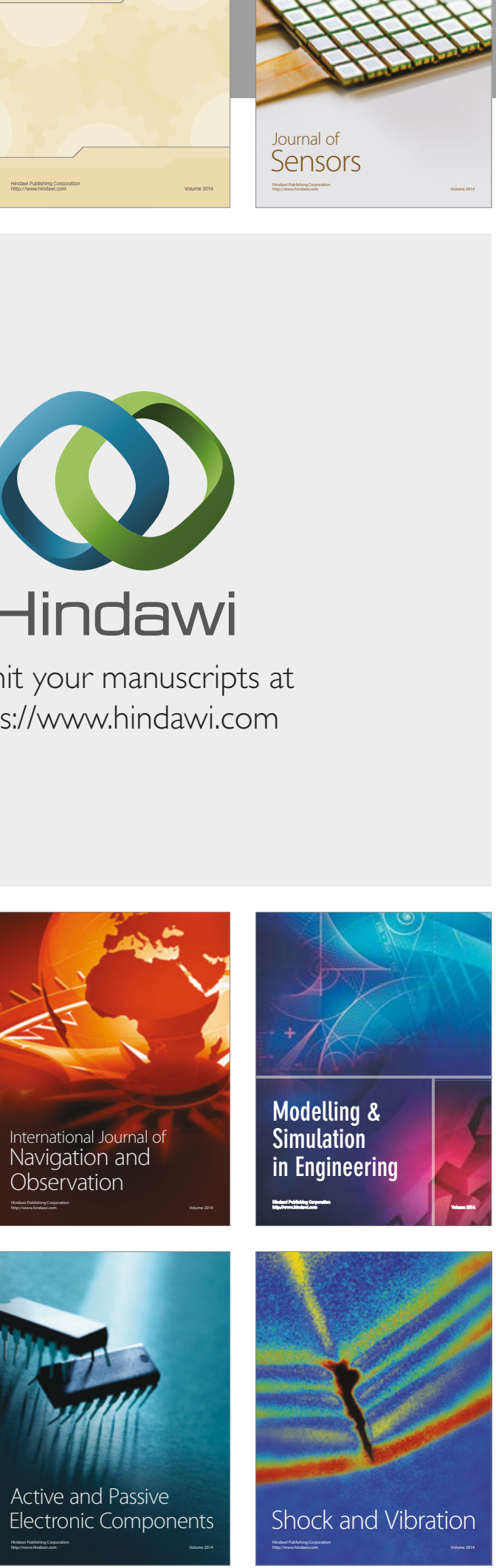
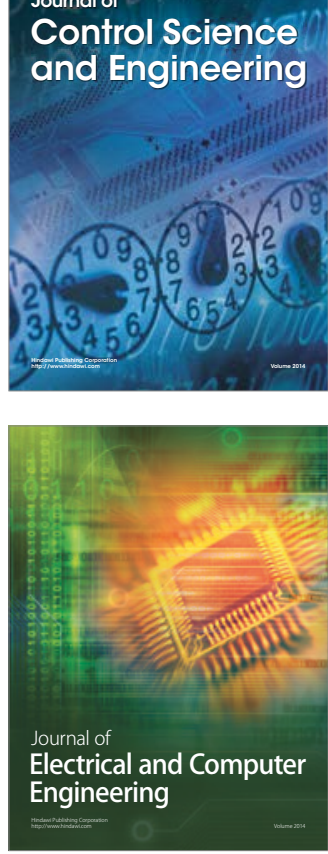

Distributed

Journal of

Control Science

and Engineering
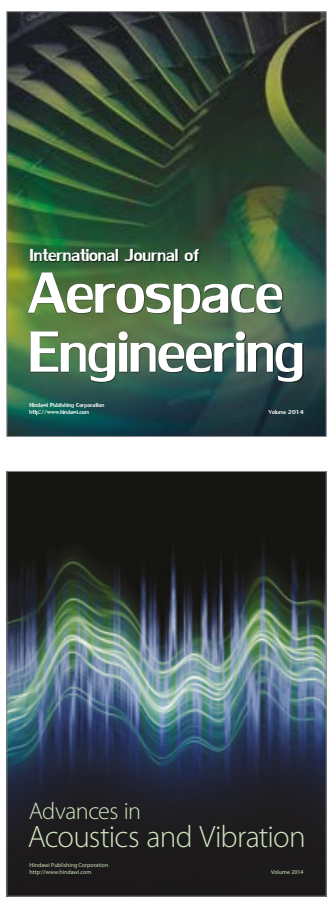

Sensor Networks 